\title{
Reflexos, reações e tônus muscular de bebês pré- termo em um programa de intervenção precoce
}

\author{
Reflexes, reactions, and muscle tone of preterm infants in an early intervention program
}

\author{
Renan Neves Urzêda ${ }^{1}$, Thalita Galdino de Oliveira' , Amanda Martins Campos ${ }^{1}$, \\ Cibelle Kayenne Martins Roberto Formiga²
}

\section{RESUMO}

Objetivo. Verificar a associação entre a evolução do tônus muscular, a idade de desaparecimento dos reflexos primitivos e aparecimento das reações posturais de bebês nascidos pré-termo. Método. Foram realizadas avaliações neuropsicomotoras mensais em 30 bebês pré-termo, admitidos no setor de Estimulação Precoce do "Follow-up do Bebê de Risco" do Instituto de Saúde Elpídio de Almeida, durante 18 meses após nascimento. Foi realizada correlação de Spearman. Resultados. Observou-se uma tendência de normalização do tônus muscular de acordo com o avanço da idade cronológica. A média de desaparecimento de alguns reflexos se encontrava fora da faixa de normalidade. Observou-se correlação direta negativa entre tônus muscular e reflexos primitivos e inversa entre tônus e reações posturais, ou seja, em geral, na medida em que o tônus muscular se normalizava (diminuía), desapareciam os reflexos primitivos e se desenvolviam (aumentavam) as reações posturais. Conclusão. Houve uma tendência de normalização do tônus muscular de acordo com o avanço da idade cronológica. A aquisição das reações posturais não foi diretamente prejudicada pela persistência de alguns reflexos primitivos devido a normalização do tônus muscular e pela facilitação dos movimentos ativos do bebê através dos estímulos oferecidos pelo programa de intervenção precoce.

Unitermos. Recém-nascido prematuro, Desenvolvimento musculoesquelético, Desenvolvimento infantil, Intervenção precoce. Citação. Urzêda RN, Oliveira TG, Campos AM, Formiga CKMR. Reflexos, reações e tônus muscular de bebês pré-termo em um programa de intervenção precoce.

Trabalho realizado no Instituto de Saúde Elpídio de Almeida (ISEA), Campina Grande-PB, Brasil.

1.Acadêmicos do Curso de Fisioterapia da Universidade Estadual de Goiás (UEG), Goiânia-GO, Brasil.

2.Fisioterapeuta, Doutora em Ciências Médicas pela FMRP/USP, Professora do Curso de Fisioterapia da UEG, Goiânia-GO, Brasil.

\section{SUMMARY}

Objective. To study the association between changes in muscle tone, age of disappearance of primitive reflexes, and the appearance of postural reactions of infants born preterm. Method. Neuropsychomotor assessments were evaluated monthly in 30 preterm infants admitted in the sector of Early Intervention of "Follow-up of High Risk Infants" at Health Institute Elpidio de Almeida, to 18 months after birth. It was performed Spearman correlation. Results. There was a tendency for normalization of muscle tone in accordance with the advance of chronological age. The mean time of disappearance of some reflections was outside the normal range. There was direct correlation between muscle tone and primitive reflexes, and inverse correlation between tone and postural reactions, that is, in general, in that muscle tone is normalized (reduced), primitive reflexes disappear and developed (increased) reactions posture. Conclusion. There was a tendency for normalization of muscle tone in accordance with the advance of chronological age. The acquisition of postural reactions was not directly affected by the persistence of some primitive reflexes due to normalization of muscle tone and the facilitation of active movement of the infant through the stimulus provided by an early intervention program.

Keywords. Premature, Musculoskeletal development, Child Development, Early intervention.

Citation. Urzêda RN, Oliveira TG, Campos AM, Formiga CKMR. Reflexes, reactions, and muscle tone of preterm infants in an early intervention program.
Endereço para Correspondência: Cibelle KMR Formiga R. Mamoré, Quadra F6, Lote 12, Residencial Araguaia CEP 74883-015, Goiânia-GO, Brasil. E-mail: cibellekayenne@gmail.com

Artigo Original Recebido em: 08/03/2009 Revisado em: 09/03/2009 a 14/11/2009

Aceito em: 15/11/2009 Conflito de interesses: não 


\section{INTRODUÇÃO}

O desenvolvimento neuropsicomotor caracteriza-se por uma seqüência de mudanças que ocorrem no comportamento da criança com a idade e pode sofrer influências biológicas e ambientais'.

Alguns fatores podem gerar riscos para o desenvolvimento da criança, podendo ocorrer em qualquer fase, ou seja, pré, peri e pós-natal². A sua incidência dependerá das características maternas, da gestação e de acordo com as influências ambientais, sociais e econômicas ${ }^{3}$. Quanto maior o efeito acumulativo de fatores de risco, maiores serão as chances de a criança desenvolver-se de maneira mais lenta, quando comparada a outras da mesma faixa etária ${ }^{4,5}$.

De acordo com a Organização Mundial de Saúde (OMS), a criança é classificada como prematura quando nasce com menos de 37 semanas de gestação ${ }^{6}$. Sendo assim, o nascimento prematuro representa uma agressão ao feto, uma vez que, em sua última etapa intra-uterina, esse ainda apresenta órgãos em fase de desenvolvimento, com imaturidade morfológica e funcional. Em decorrência principalmente da imaturidade do SNC, bebês nascidos pré-termo apresentam maior chance de desenvolver alterações no seu desenvolvimento ${ }^{7}$.

O bebê pré-termo apresenta diferenças anatômicas e fisiológicas quando comparado ao bebê nascido a termo. O SNC no recém-nascido pré-termo se encontra em uma fase de desenvolvimento diferente daquele do recém-nascido a termo, estando em processo de maturação (aparecimento de sulcos e giros do encéfalo, migração celular, mielinização, arborização dendrítica e das terminações axonais) sob condições não fisiológicas e frequentemente adversas, levando a uma mudança no padrão de comportamento neurológico do prematuro ${ }^{8}$.

Os avanços científicos na área da Neonatologia que ocorreram nas últimas décadas, especialmente a implantação da Unidade de Terapia Intensiva (UTI) neonatal, contribuíram para o aumento das taxas de sobrevivência dos lactentes prematuros $^{9-11}$. Isso propiciou um aumento considerável dos limites da viabilidade desses bebês, visto que, atualmente, recém-nascidos com idades gestacionais cada vez menores recebem alta das unidades de cuidados neonatais em condições aparentemente satisfatórias ${ }^{10}$.
Embora a mortalidade dos bebês de alto risco tenha diminuído, houve um aumento na incidência de distúrbios neuropsicomotores entre os bebês sobreviventes. Esse fato tem motivado a realização de inúmeras pesquisas cientificas na área, que visam, através do acompanhamento dessas crianças ao longo do tempo, esclarecer o que realmente ocorre no desenvolvimento dessa população e determinar os possíveis fatores de risco envolvidos ${ }^{12-14}$.

A maior parte dos movimentos do recémnascido é representada por reflexos primitivos, que vão desaparecendo durante os seis primeiros meses de vida, quando estruturas neurológicas hierarquicamente mais recentes (corticais) vão amadurecendo e tornando-se funcionais ${ }^{15}$. Entre os reflexos, destacam-se: sucção, Moro, preensão palmar, preensão plantar, Galant, marcha, reflexo tônico cervical assimétrico (RTCA) e anfíbio. A ausência desses reflexos em idades em que deveriam estar presentes ou a persistência desses em idade em que deveriam ter desaparecido, poderá indicar prejuízo neurológico ${ }^{16}$.

A avaliação neurológica do bebê é composta basicamente pela observação do tônus muscular, da postura, da movimentação espontânea e reflexos primitivos. Sob este aspecto ressalta-se a importância da identificação precoce de desvios do desenvolvimento, de forma que se possa intervir em uma fase em que ainda não existam padrões patológicos instalados e em que a plasticidade cerebral ainda é grande, tornando os resultados mais rápidos e efetivos ${ }^{17}$.

A intervenção precoce é definida como o conjunto de ações tendentes a proporcionar à criança as experiências sensório-motoras de que esta necessita desde o seu nascimento, para desenvolver, ao máximo, seu potencial neuropsicomotor. Isso se alcança através da presença de pessoas e objetos, em quantidade e oportunidades adequadas e em um contexto de situações de variada complexidade, que geram na criança certo interesse; e atividades, condições necessárias para lograr uma reação dinâmica com seu meio ambiente e uma aprendizagem efetiva ${ }^{18}$.

Os objetivos do presente estudo foram analisar a associação entre a evolução do tônus muscular, a idade de desaparecimento dos reflexos primitivos e aparecimento das reações posturais de bebês nascidos pré-termo participantes de um 
programa de intervenção precoce de 1 a 18 meses de idade corrigida.

\section{MÉTODO}

\section{Amostra}

Participaram do estudo uma amostra de 30 bebês pré-termo, de ambos os sexos, admitidos no setor de Estimulação Precoce do "Follow-up do Bebê de Risco" do Instituto de Saúde Elpídio de Almeida (ISEA), na cidade de Campina Grande-PB.

Os critérios de inclusão foram: bebês prematuros (idade gestacional $<37$ semanas, de acordo com a OMS), e que apresentaram uma ou mais das complicações típicas da prematuridade, tais como baixo peso ao nascer, hiperbilirrubinemia, problemas respiratórios e distúrbios metabólicos.

Foram excluídos do estudo os bebês que apresentaram outras alterações tais como mielomeningocele, síndromes genéticas, deformidades congênitas de membros, micro e macrocefalia. Os materiais utilizados para coleta de dados foram: fichas de avaliação e evolução, protocolos, gravador, fitas cassete, máquina fotográfica e computadores.

Os pais ou responsáveis pelos bebês receberam uma ampla e detalhada explicação sobre o estudo e seus objetivos, assinando o Termo de Consentimento Livre e Esclarecido para participação na pesquisa. O estudo foi aprovado pelo Comitê de Ética em Pesquisa da Universidade Federal da Paraíba (UFPB), com protocolo 123/01.

\section{Procedimentos}

Foi realizada uma avaliação fisioterapêutica, com duração média de 40 minutos, para a coleta das seguintes informações: dados da identificação do recém nascido (nome, sexo, idade cronológica, data de nascimento, nomes e idades dos pais e endereço completo); dados do nascimento (local e tipo de parto, médico pediatra, idade gestacional, Apgar, peso, altura, perímetro cefálico e torácico); história clínica da gestação; parto e pós-parto (duração, intercorrências, acompanhamento médico); e avaliação neuropsicomotora do bebê (tônus muscular, reflexos primitivos, reações de retificação e equilíbrio). Os dados referentes à avaliação neuropsicomotora foram coletados mensalmente no período de 18 meses. Todas as avaliações foram realizadas pela fisioterapeuta responsável pelo serviço (Formiga CKMR).
Após a avaliação os bebês foram incluídos no Programa de Intervenção Precoce, com acompanhamento do desenvolvimento até aproximadamente 18 meses de idade corrigida, pela mesma avaliadora. O programa de intervenção precoce consistia em atividades de estimulação sensóriomotora baseado no Conceito Neuroevolutivo Bobath e Método Samarão Brandão. Cada criança foi atendida considerando o desenvolvimento motor em que se encontrava e as técnicas de tratamento eram direcionados para a estimulação do controle postural, estímulo das coordenações sensório-motoras e transferências de postura. Os bebês realizavam em média dois atendimentos semanais com duração média de 40 minutos cada.

Para o cálculo da correção da idade, subtraiuse a idade gestacional do bebê pré-termo, de um total de 40 semanas. Esta diferença corresponde ao tempo de prematuridade, que é então descontado da idade cronológica da criança. A cada mês os bebês eram reavaliados tendo sua evolução registrada na ficha de desenvolvimento os quais deveriam obedecer aos critérios do exame inicial.

As reavaliações mensais dos bebês obedeceram aos seguintes critérios: a) avaliação do tônus muscular (tônus normal, hipertonia, hipotonia, tônus flutuante) foi realizada através da manipulação passiva dos membros superiores (MMSS) e inferiores (MMII) do bebê; b) avaliação dos reflexos e reações: verificação da presença e ausência de reflexos primitivos, reações de retificação, equilíbrio e proteção de acordo com as fases do desenvolvimento neuropsicomotor conforme preconiza o exame neurológico.

\section{Análise Estatística}

Foi realizado teste de correlação de Spearman entre tônus muscular, reflexos primitivos e reações posturais a fim de observar a associação entre o tônus muscular e a atividade reflexa no desenvolvimento neuropsicomotor dos bebês. Foi considerado um nível de significância de 5\% $(p<0,05)$.

\section{RESULTADOS}

Observou-se uma tendência de normalização do tônus muscular de acordo com o avanço da idade cronológica. No primeiro mês registrou-se uma freqüência de 21 bebês com hipertonia muscular nos quatro membros enquanto apenas um 
bebê apresentou tônus muscular normal. Já ao $18^{\circ}$ mês verificou-se uma inversão nesta relação, com 25 crianças apresentando tônus muscular normal e 4 crianças permanecendo com hipertonia muscular nos quatro membros (Figura 1).

Foi observado que a média de desaparecimento dos reflexos de preensão palmar, RTCA, Moro e marcha se encontrava fora da faixa de normalidade, por persistirem em idades em que já deveriam estar ausentes (Tabela 1).

Verificou-se que os bebês apresentaram as reações posturais em idade muito próxima da idade considerada normal levando-se em conta os padrões do desenvolvimento normal (Tabela 2).

Os valores negativos dos coeficientes de correlação encontrados demonstram uma relação inversa entre a tendência de normalização do tônus muscular e a idade de desaparecimento dos reflexos primitivos (Tabela 3). Na medida em que o tônus muscular do bebê foi sendo modulados com a idade os reflexos primitivos deixaram de ser observáveis na avaliação neurológica.

A correlação negativa verificada revela também uma relação inversa entre a normalização progressiva do tônus muscular e a idade de aparecimento das reações posturais (Tabela 4). Na medida em que o tônus muscular do bebê foi sendo moduladas com a idade as reações posturais começaram a ser observadas na avaliação neurológica.
Tabela 1. Análise da persistência dos reflexos primitivos em um grupo de bebês pré-termos (em meses).

\begin{tabular}{|c|c|c|c|c|}
\hline $\begin{array}{c}\text { Reflexo } \\
\text { Avaliado }\end{array}$ & Média & DP & Mínimo & Máximo \\
\hline Prensão palmar & 7,5 & 3 & 3 & 18 \\
\hline Prensão plantar & 10,5 & 3 & 2 & 17 \\
\hline RTCA & 6 & 3 & 3 & 18 \\
\hline Moro & 6,5 & 3 & 1 & 18 \\
\hline Marcha & 5 & 2,5 & 1 & 10 \\
\hline Galant & 3 & 2 & 1 & 10 \\
\hline Anfíbio & 6,5 & 2 & 1 & 10 \\
\hline $\begin{array}{c}\text { Reação positiva } \\
\text { de suporte }\end{array}$ & 9,5 & 4 & 2 & 18 \\
\hline
\end{tabular}

RTCA=reflexo tônico cervical assimétrico; $D P=$ desvio padrão.

\section{DISCUSSÃO}

Os resultados do presente estudo permitiram verificar a ocorrência dos reflexos primitivos e das reações posturais associados com a modulação do tônus muscular nos 18 primeiros meses de vida de bebês nascidos pré-termo, havendo uma correlação direta entre o tônus muscular e reflexos primitivos (a redução do tônus associou-se a redução dos reflexos primitivos), e uma correlação inversa entre tônus muscular e reações posturais (a redução do tônus associou-se com aumento das reações posturais).

Figura 1. Evolução do tônus muscular das crianças do $1^{\circ}$ ao $18^{\circ}$ mês de idade corrigida.

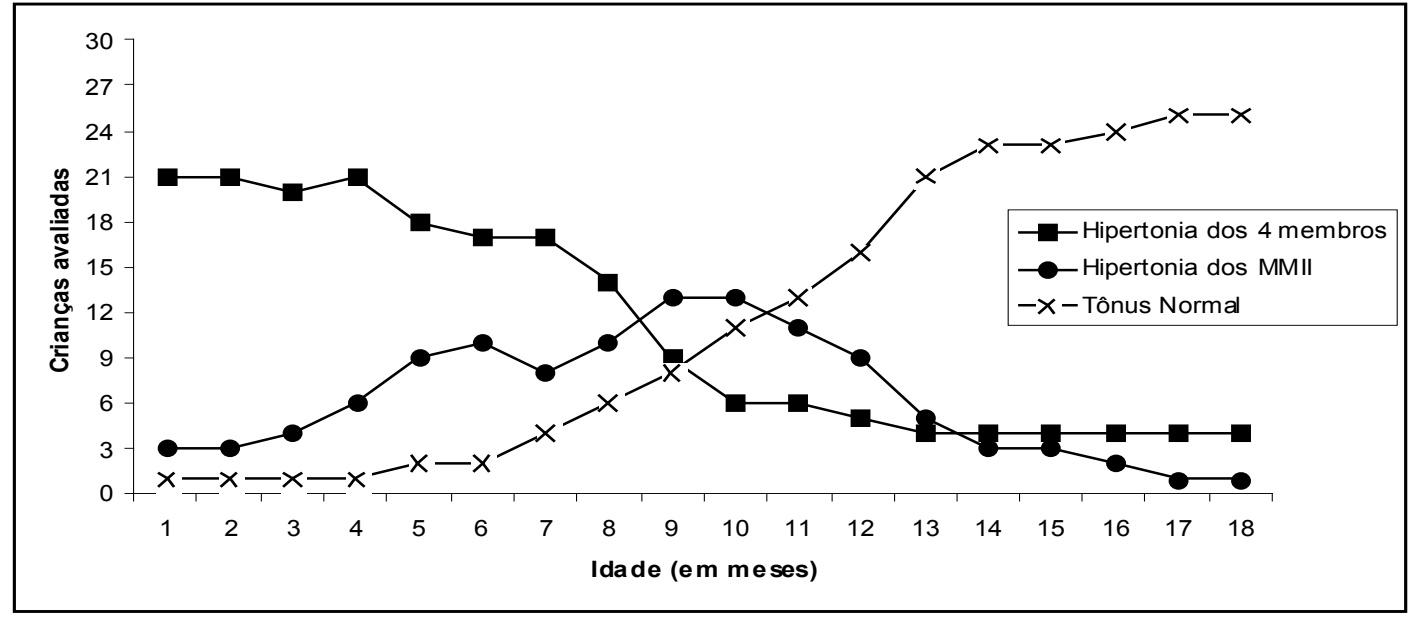

MMII=membros inferiores. 
Tabela 2. Período de aquisição das reações posturais em um grupo de bebês pré-termo (em meses).

\begin{tabular}{|c|c|c|c|c|}
\hline $\begin{array}{c}\text { Reação } \\
\text { postural avaliada }\end{array}$ & Média & DP & Mínimo & Máximo \\
\hline Retificação cervical & 5,5 & 1,5 & 2 & 10 \\
\hline Retificação corporal & 5,5 & 1,5 & 3 & 10 \\
\hline $\begin{array}{c}\text { Retificação } \\
\text { labiríntica }\end{array}$ & 4,5 & 2 & 1 & 10 \\
\hline Retificação óptica & 4 & 2,5 & 1 & 12 \\
\hline Proteção para frente & 7 & 2 & 4 & 12 \\
\hline $\begin{array}{c}\text { Proteção } \\
\text { para os lados }\end{array}$ & 9 & 2,5 & 6 & 18 \\
\hline Proteção para trás & 11 & 2,5 & 7 & 18 \\
\hline Landau I & 6,5 & 2 & 4 & 12 \\
\hline Landau II & 9,5 & 2 & 6 & 18 \\
\hline Equilíbrio sentado & 9 & 2 & 6 & 16 \\
\hline Equilíbrio de gato & 11 & 2,5 & 8 & 18 \\
\hline Equilíbrio de joelho & 11 & 3,5 & 8 & 18 \\
\hline Equilíbrio de pé & 13,3 & 3,5 & 10 & 18 \\
\hline
\end{tabular}

$\mathrm{DP}=$ desvio-padrão.

Tabela 3. Análise da correlação entre tônus muscular e reflexos primitivos

\begin{tabular}{|c|c|c|c|}
\hline $\begin{array}{c}\text { Tônus } \\
\text { muscular }\end{array}$ & $\begin{array}{c}\text { Reflexos } \\
\text { primitivos }\end{array}$ & $\begin{array}{c}\text { Coeficiente de } \\
\text { Correlação }(r)\end{array}$ & Valor de $p$ \\
\hline $5^{\circ}$ ao $6^{\circ}$ mês & RTCA & $(-0,42) \leq r \leq(-0,35)$ & $\leq 0,05$ \\
\hline $8^{\circ}$ mês & $\begin{array}{c}\text { Reflexo de } \\
\text { Moro }\end{array}$ & $r=(-0,41)$ & $\leq 0,03$ \\
\hline $7^{\circ}$ ao $10^{\circ}$ mês & $\begin{array}{c}\text { Reação } \\
\text { positiva de } \\
\text { suporte }\end{array}$ & $(-0,5) \leq r \leq(-0,47)$ & $\leq 0,03$ \\
\hline
\end{tabular}

RTCA=reflexo tônico cervical assimétrico.
Estes achados são condizentes com a literatura, no que se refere à evolução normal do tônus muscular no decorrer dos meses. Após o parto, o recém nascido costuma apresentar tônus muscular aumentado, denominado "hipertonia fisiológica", que se manterá durante os meses subseqüentes, sofrendo uma progressiva queda no final do terceiro mês, que então deverá estabilizar-se após o primeiro ano de vida ${ }^{19}$. Além disso, o ambiente extra-uterino apresenta características físicas que influenciam o desenvolvimento das habilidades motoras do bebê, tais como, a gravidade, a postura corporal, a superfície de apoio e efeitos de estimulação terapêutica ${ }^{20}$.

Há autores que defendem que anormalidades no tônus muscular são um dos sinais indicativos de alterações no desenvolvimento neurosensório-motor ${ }^{21}$. Com relação às crianças que apresentaram persistência da hipertonia muscular, podemos destacar que esta deve ser considerada como um sinal de alerta para possíveis lesões centrais nos primeiros meses de vida extra-uterina ${ }^{18,22}$. Nesse sentido, a intervenção realizada nos primeiros meses de vida atuaria na modulação do tônus muscular, favorecendo as habilidades motoras do bebê ${ }^{17}$.

O RTCA é um dos sinais indicativos de atraso na maturação do sistema nervoso quando presente em um grau elevado, em qualquer idade, mas principalmente após o $7^{\circ}$ mês de vida. Quando persistente este sinal impedirá que a cabeça se movimente para o lado oposto a que estiver rodada, impossibilitando assim a aquisição das coordenações sensório-motoras primárias, tais como, a viso-cefálica, áudio-cefálica e mão-mão ${ }^{22-24}$.

Tabela 4. Análise da correlação entre tônus muscular e reações posturais

\begin{tabular}{|c|c|c|c|}
\hline Tônus muscular & Reaçães posturais & Coeficiente de Correlação $(r)$ & Valor de $p$ \\
\hline $9^{\circ}$ ao $18^{\circ}$ mês & Reação de retificação corporal & $(-0,56) \leq r \leq(-0,38)$ & $\leq 0,04$ \\
\hline $17^{\circ}$ ao $18^{\circ}$ mês & Reação de retificação labiríntica & $r=(-0,39)$ & $\leq 0,03$ \\
\hline $18^{\circ}$ mês & Reação retificação óptica & $r=(-0,39)$ & $\leq 0,03$ \\
\hline $7^{\circ}$ ao $12^{\circ}$ mês & Reação de proteção para frente & $(-0,45) \leq r \leq(-0,35)$ & $\leq 0,04$ \\
\hline $7^{\circ}$ ao $16^{\circ}$ mês & Reação de proteção para os lados & $(-0,5) \leq r \leq(-0,35)$ & $\leq 0,05$ \\
\hline $12^{\circ}$ ao $15^{\circ}$ mês & Reação de proteção para trás & $(-0,47) \leq r \leq(-0,39)$ & $\leq 0,04$ \\
\hline $7^{\circ}$ ao $12^{\circ}$ mês & Reação de Landau I & $(-0,6) \leq r \leq(-0,35)$ & $\leq 0,05$ \\
\hline $7^{\circ}$ ao $18^{\circ}$ mês & Reação de Landau II & $(-0,6) \leq r \leq(-0,3)$ & $\leq 0,05$ \\
\hline $6^{\circ}$ ao $15^{\circ}$ mês & Reação de equilíbrio sentado & $(-0,55) \leq r \leq(-0,35)$ & $\leq 0,05$ \\
\hline $9^{\circ}$ mês & Reação de equilíbrio de gato & $r=(-0,43)$ & $\leq 0,03$ \\
\hline $7^{\circ}$ ao $15^{\circ}$ mês & Reação de equilíbrio de pé & $(-0,55) \leq r \leq(-0,41)$ & $\leq 0,03$ \\
\hline
\end{tabular}


A permanência do reflexo de preensão palmar e do reflexo de Moro após o $6^{\circ}$ mês de vida ${ }^{25,26}$, e do reflexo de marcha automática além do $2^{\circ}$ ou $3^{\circ}$ mês de vida ${ }^{24,27}$ são considerados sinais precoces de suspeita de lesão neurológica. Entretanto, alguns autores afirmam que o reflexo de marcha nunca desaparece no desenvolvimento e a sua observação é menos evidente a partir do $3^{\circ}$ mês devido ao aumento da massa muscular dos membros inferiores do bebêt $\hat{e}^{28,29}$.

O desenvolvimento de padrões motores processa-se seqüencialmente de acordo com o repertório motor humano, porém não necessariamente da mesma forma em todos os indivíduos, isso se dá devido a diferenças especificas e intrínsecas de cada um, o que possibilita uma visão mais abrangente sobre o desenvolvimento neuropsicomotor ${ }^{6}$.

O desenvolvimento atrasado ou anormal das reações posturais e de equilíbrio reflete a presença de distúrbios neurológicos, como as encefalopatias crônicas não evolutivas ${ }^{30}$. Após o nascimento, ou ainda no primeiro trimestre de vida, já é possível identificar lesões cerebrais graves a partir das anormalidades presentes no tônus muscular, nos reflexos primitivos ausentes ou exacerbados e nas reações posturais ${ }^{31}$.

Os resultados encontrados no presente estudo ratificam a necessidade de identificar precocemente os sinais de atrasos do desenvolvimento de bebês vulneráveis do ponto de vista biológico, possibilitando o encaminhamento e a realização de um programa de intervenção precoce. As técnicas e manuseios utilizados na intervenção destinam-se a facilitar o desenvolvimento das coordenações sensório-motoras do bebê e potencializar as capacidades da criança. $O$ tratamento também objetiva orientar pais e/ou cuidadores para melhor adaptação aos cuidados oferecidos ao bebê, além de posicionamentos e estímulos em casa ${ }^{20}$.

\section{CONCLUSÃO}

O estudo verificou que houve uma tendência de normalização do tônus muscular de acordo com o avanço da idade cronológica. A aquisição das reações posturais não foi diretamente prejudicada pela persistência de alguns reflexos primitivos além da idade considerada normal. Justifica-se este resultado em função da normalização do tônus muscular ao longo dos meses e da facilitação dos movimentos ativos do bebê através dos estímulos oferecidos pelo programa de intervenção precoce. Ressalta-se que os resultados encontrados restringem-se à amostra estudada e deve-se ter cautela na generalização destes.

\section{REFERÊNCIAS}

1.Gallahue DL, Ozmun JC. Compreendendo o Desenvolvimento Motor: Bebês, crianças, adolescentes e adultos. São Paulo: Phorte Editora, 2003, 641p.

2.Shermont AG, Cunha MS, Sales LMN, Moraes AN, Malveira SS. Avaliação do desenvolvimento pela escala de Denver II, de recém nascidos prematuros de baixo peso. Rev Para Med 2005; 19:59-66.

3.Sapienza G, Predomônico MRM. Risco, proteção e resiliência no desenvolvimento da criança e do adolescente. Psicol Est 2005;10:209-16.

4.Halpern R, Giugliani E, Victora C, Barros F, Horta B. Fatores de risco para suspeita de atraso no desenvolvimento neuropsicomotor aos 12 meses de vida. J Pediatr 2000;76:421-8.

5.Salles RF. Análise de um programa de intervenção com bebês e famílias atendidas em unidades básicas de saúde - SUS (Tese). São Paulo: UFSCar, 2000, 158p.

6.Marcondes E. Pediatria Básica. São Paulo: Sarvier, 2002, 843p. 7.Scochi CGS, Kokuday NLP, Riul NJS, Rossanez LSS, Fonseca LNM, Leite AM. Incentivando o vínculo mãe-filho em situação de prematuridade: As intervenções de enfermagem no Hospitas das Clínicas de Ribeirão Preto. Rev Lat Am Enfer 2003;11:539-43.

8.Moreira MEL, Rodrigues MA. O Bebê na UTI: Intercorrências e Exames Mais Freqüentes. In: Moreira MEL, Braga NA, Morsch DE. Quando a Vida Começa Diferente: O Bebê e sua Família na UTI Neonatal. Rio de Janeiro: Fiocruz, 2003, p.43-50.

9.Rugolo LMSS. Crescimento e desenvolvimento a longo prazo do prematuro extremo. J Pediatr 2005;81:101-10.

10.Resegue R, Puccini RF, Silva EMK. Fatores de Risco Associados a Alterações no Desenvolvimento da Criança. J Pediatr 2007;29:117-28.

11.Santos RS, Araújo AP, Porto MA. Early Diagnosis of Abnormal Development of Preterm Newborns: Assessment Instruments. J Pediatr 2008;84:289-99.

12.Perlman MJ. Neurobehavioral Deficits in Premature Graduates of Intensive Care - Pontential Medical and Environmental Risk Factors. J Pediatr 2001;108:1339-48.

13.Mancini MC, Teixeira S, Araújo LG, Paixão ML, Magalhães LC, Coelho ZAC, et al. Estudo do Desenvolvimento da Função Motora aos 8 e 12 Meses de Idade em Crianças Nascidas Prétermo e a Termo. Arqneuropsiquiatr 2002;60:974-80.

14.Rosa-Neto F, Caon G, Bissani C, Silva C, Sousa M, Silva E. Características Neuropsicomotoras de Crianças de Alto Risco Neurológico Atendidas em um Programa de Follow up. Pediatr Mod 2005;42:79-85.

15.Vilanova LCP. Aspectos neurológicos do desenvolvimento do comportamento da criança. Rev Neurocienc 1998;6:10610.

16.Scherzer AL. Diagnostic approach to the infant. In: Scherzer AL. Early diagnosis and interventional therapy in cerebral palsy. New York: Marcel Dekker, 2000, p.49-94. 
17.Marlow N. Neurocognitive outafter very preterm birth Arch Dis Child Fetal Neonatal Ed 2004;89:224-8.

18.Brandão JS. Bases do tratamento por estimulação precoce da paralisia cerebral, ou, dismotria cerebral ontogenética. São Paulo: Memnon, 1992, 104p.

19.Schoen ACB, Ricci E, Oliveira WG. Aplicação do prognóstico de deambulação em crianças com paralisia cerebral descrito por Souza e Ferraretto. Arq Cienc Saúde Unipar 2003;7:51-6. 20.Formiga CKMR. Programa de intervenção precoce com bebês pré -termo e suas famílias: subsídios para prevenção de deficiências (Tese). São Paulo: UFSCar, 2003, 222p.

21.Guimarães EL. Estudo para detecção precoce de sinais indicativos de alterações no desenvolvimento neuro-sensóriomotor em bebês de risco (Tese). São Paulo: UFSCar, 2001, 159 p.

22.Bobath B, Bobath KA. Diferenciação entre padrões primitivos e anormais. In: Bobath B. Desenvolvimento motor nos diferentes tipos de paralisia cerebral. São Paulo: Manole, 1990. p.19-22.

23.Flehmig I. Texto e atlas do desenvolvimento normal e seus desvios no lactente - Diagnóstico e Tratamento Precoce do Nascimento até o $18^{\circ}$ mês. Rio de Janeiro: Editora Atheneu, 2005, 316p.
24.Levitt S. O tratamento da paralisia cerebral e do retardo motor. São Paulo: Editora Manole, 2001, 286p.

25.Volpe JJ. Neurology of Newborn. Pliladelphia: Saunders, $1987,876 \mathrm{p}$.

26.Capute AJ, Shapiro BK, Accardo PJ, Wachtel RC, Ross A, Palmer FB. Motor Functions Associated Primitive Reflex Profiles. Dev Med Child Neurol 1982;24:662-9.

27.Figueiredo HB. Diagnóstico precoce da paralisia cerebral. In: Leitão A. Paralisia cerebral: diagnóstico, terapia, reabilitação. Rio de Janeiro: Atheneu, 1983, p.15-29.

28.Shepherd RB. Fisioterapia em Pediatria. São Paulo: Editora Santos, 1995, 21 p.

29.Thelen E. Motor Development: A New Synthesis. Am Psychol 1995;50:79-95.

30.Guimarães EL, Tudella E. Reflexos Primitivos e Reações Posturais como Sinais Indicativos de Alterações Neuro-SensórioMotoras em Bebês de Risco. J Pediatr 2003;25:28-35.

31.Piper MC, Darrah J, Byrne P. Impact of gestational age on preterm motor development at 4 months chronological and adjusted ages. Child Care Health Dev 1989;15:105-15. 